\title{
A etnografia como método de pesquisa em Informática na Educação: Revisão Sistemática de \\ Literatura
}

\section{The ethnography as the research method in Computer Science in Education: Systematic Literature Review}

SCHEILA WESLEY MARTINS

Pontifícia Universidade Católica de Minas (PUC-Minas)

DANIEL EUGÊNIO NEVES Pontifícia Universidade Católica de Minas (PUC-Minas)

MÔNICA DA CONSOLAÇÃO MACHADO Pontifícia Universidade Católica de Minas (PUC-Minas)

EZEQUIEL MENDES DUQUE Pontifícia Universidade Católica de Minas (PUC-Minas)

ARTUR MARTINS MOL Pontifícia Universidade Católica de Minas (PUC-Minas)

LUCILA ISHITANI Pontifícia Universidade Católica de Minas (PUC-Minas)

\begin{abstract}
Resumo: Etnografia é um método de pesquisa qualitativa pouco citado em pesquisas na área de informática na educação no Brasil. Com o objetivo de demonstrar o potencial de utilização desse método e sua aplicação para a comunidade científica brasileira da área, foi realizada uma Revisão Sistemática da Literatura (RSL) na base da Association for Computing Machinery (ACM), avaliando artigos publicados entre 2015 e 2016. O método etnográfico é muito relevante para pesquisas em que o foco está no fator humano, inclusive pesquisas em educação. Assim, consideramos que os resultados deste trabalho podem contribuir para que grupos de pesquisa da área no Brasil conheçam a aplicação do método no contexto internacional, analisando a assertividade da abordagem etnográfica afim de incentivar sua apropriação pelos pesquisadores área de Informática na Educação, amplificando a confiabilidade dos resultados de suas pesquisas, e, por consequência, beneficiando a prática educacional em seus vários níveis e modalidades.
\end{abstract}

Palavras-chave: Etnografia. Revisão Sistemática de Literatura. Pesquisa Qualitativa. Informática na Educação. 


\begin{abstract}
Ethnography is a qualitative research method, little applied in the Computer Science in Education domain in Brazil. In order to demonstrate the potential of this method and its application to the Brazilian community, a Systematic Literature Review (SLR) was carried out at the Association for Computing Machinery (ACM) database, evaluating articles published between 2015 and 2016 The ethnographic method is very relevant for research that focuses on the human factor, including research in education. Thus, we consider the results of this work can contribute with research groups in the area in Brazil to know the application of the method in the international context, analyzing the assertiveness of the ethnographic approach in order to encourage its appropriation by researchers in the field, amplifying the reliability of research results, and consequently, benefiting the educational practice in its various levels and modalities.
\end{abstract}

Keywords: Ethnography. Systematic Literature Review. Qualitative research. Computer Science in Education. 


\section{Introdução}

Etnografia é um método de pesquisa qualitativa, historicamente muito aplicado nas áreas de ciências sociais, humanas e da saúde. Considerando a importância desse método para pesquisas que tenham por objetivo compreender o relacionamento das pessoas com a tecnologia, como ocorre em muitas pesquisas da área de Informática na Educação, pondera-se que esse seria um indicativo da necessidade da comunidade da área de computação no Brasil conhecer melhor da etnografia para poder utilizá-lo com mais frequência.

Para auxiliar nesse propósito, foi realizada uma Revisão Sistemática da Literatura (RSL) (KITCHENHAM, 2007), pesquisando pelos termos-chave ethnograp* e (education ou learning) em repositórios nacionais e internacionais. A partir da análise de 35 artigos selecionados, foi possível extrair orientações sobre: o objetivo de pesquisas que utilizaram etnografia, o número de participantes nas pesquisas, quanto tempo durava a aplicação do método, como a coleta e a análise de dados foram realizadas. A avaliação desse conjunto de informações pode auxiliar os pesquisadores brasileiros da área de Informática na Educação no planejamento metodológico de suas pesquisas, contribuindo, dessa forma, para trazer mais segurança para a utilização da abordagem etnográfica, e possibilita assegurar maior confiabilidade aos resultados de seu trabalho.

Este artigo está organizado da seguinte forma: a Seção 2 aborda uma visão geral sobre o método de pesquisa etnográfico; a Seção 3 apresenta em detalhes o processo de pesquisa adotado neste estudo, bem como os elementos focais da presente revisão sistemática; a Seção 4 apresenta um resumo quantitativo dos resultados obtidos, respondendo às questões de pesquisa estipuladas; a Seção 5 faz uma reflexão sobre alguns aspectos relevantes identificados por este estudo e, por fim, a Seção 6 demonstra as principais conclusões sobre os resultados obtidos.

\section{Etnografia}

Segundo Hammersley e Atkinson (2007) e Creswell (2002), a etnografia é compreendida como um dos métodos qualitativos de pesquisa cuja definição e metodologia podem se sobrepor a uma extensiva relação de abordagens de pesquisa, que usualmente são bastante empregadas nas pesquisas em ciência social e em antropologia.

A concepção tradicional da etnografia se desenvolveu em paralelo com outro conceito essencial da pesquisa antropológica, a etnologia (HAMMERSLEY; ATKINSON, 2007), baseada na extração de trechos dos diários de bordo dos navegadores europeus, os quais relatavam as descobertas feitas em suas expedições ao novo mundo. A prática etnológica pura possibilitou a descrição detalhada das terras conquistadas, sem, contudo, estabelecer uma correlação entre o campo territorial e a população local. Já a etnografia definiu uma abordagem metodológica teórica, na qual a investigação empírica se encontra com a análise comparativa de forma equilibrada, amplificando a profundidade e a confiabilidade da pesquisa qualitativa, que foi possibilitada pela etnologia (HAMMERSLEY; ATKINSON, 2007; CRESWELL, 2002). 
O estudo etnográfico se ocupa de um projeto qualitativo no qual os pesquisadores tentam definir as similaridades e/ou as diferenças existentes na identidade cultural que descreveria uma comunidade ou um grupo não muito pequeno de indivíduos (HAMMERSLEY; ATKINSON, 2007). Os pesquisadores irão focar em conhecer a fundo os valores, crenças, comportamentos e a linguagem compartilhada pela comunidade. Esse nível de detalhamento de um domínio cultural depende muito de pesquisa de campo, baseada em intensiva e extensiva atividade de coleta de dados. As condições de acesso detalhado às informações em estudos etnográficos são providas por meio de uma diversidade de ferramentas da pesquisa qualitativa, especialmente, entrevistas e anotações de observação. É bem comum que os pesquisadores conduzam entrevistas e se utilizem de diários como forma de aumentar a quantidade e o detalhamento das informações em que se apoiam para descrever as complexidades de comportamento (CRESWELL, 2002).

Atualmente, a etnografia tem sido aplicada em diferentes domínios de pesquisa para além da antropologia, o que inclui psicologia, tecnologia, marketing, engenharia e design. Percebe-se que a abordagem etnográfica será particularmente interessante, e até mesmo recomendável, para aqueles estudos nos quais pessoas (e como elas reagem) têm significativa influência e/ou desenvolvem papel-chave para os resultados de pesquisa esperados (CRESWELL, 2002).

\section{Método}

Apesar de alguns autores afirmarem que a etnografia não é um método amplamente utilizado nas pesquisas da área de computação, ao se pesquisar preliminarmente pelo termo ethnograph* (ou seja, ethnography e outros termos relacionados, tais como ethnographic ou ethnographer) nas bases da ACM (Association for Computing Machinery), da IEEE (Institute of Electrical and Electronics Engineers) e nos artigos da área de computação da Science Direct, foram retornados, em setembro de 2016, respectivamente 2.686, 196 e 517 artigos publicados a partir de 2011.

Por outro lado, o termo não foi encontrado em artigos completos na busca realizada em repositórios de pesquisa nacionais como a Revista Brasileira de Informática na Educação (RBIE). Nos anais do Simpósio Brasileiro de Informática na Educação (SBIE), digitalmente disponíveis, foi encontrado um único artigo, datado de 2009, que citava o método (REIS \& LINHARES, 2009). Nos anais do Workshop de Informática na Escola (WIE), disponíveis digitalmente desde 2003, foram encontrados apenas três artigos que se basearam no método para realização da pesquisa: um datado de 2004 (CASAS, 2004), outro de 2010 (VENTURA \& DE CASTRO FILHO, 2010) e mais um de 2011 (ALBUQUERQUE, FERNANDES, \& CASTRO FILHO, 2011). Mesmo após a atualização da pesquisa, o artigo datado de 2016 (TIMMERMANN \& GONZÁLEZ, 2016) publicado na Anais do Workshop do Congresso Brasileiro de Informática na Educação (CIEB 2016) não pode ser incluído na presente análise devido aos critérios de exclusão adotados pelo grupo, que serão detalhados a seguir

Com o objetivo de identificar como o método de pesquisa etnográfica tem sido aplicada nas pesquisas da área de Informática na Educação no contexto internacional, foi realizada uma Revisão Sistemática de Literatura (RSL) (KITCHENHAM, 2007). Para o presente estudo, foi definida como questão principal de pesquisa (QP) "Como a etnografia foi aplicada nas pesquisas 
da área de Informática na Educação?", e também seis questões específicas (QE): "1.Quais são os objetivos dessas pesquisas?", "2.Qual era o tamanho da amostra?, "3.Qual foi a duração das pesquisas?", "4.Quais foram os procedimentos adotados para coleta de dados?", "5.Quais foram os instrumentos de coleta de dados adotados?" e, "6.Quais foram os métodos de análise de dados adotados?".

A string de pesquisa utilizada foi (ethnograp* AND [education OR learning]). Inicialmente, foi planejado realizar a pesquisa pelos termos no título, resumo e palavras-chave de artigos das bases da ACM, IEEE e Science Direct, publicados nos últimos dez anos. Após um teste piloto, identificou-se que os resultados obtidos não incluíam todos os artigos importantes já conhecidos, porque nem sempre os termos pesquisado apareciam nos campos de pesquisa definidos. Além disso, o número de resultados retornados nas três bases era demasiado elevado e tornaria a análise inviável considerando o tamanho do grupo de trabalho e o tempo estimado para desenvolvimento da pesquisa e divulgação de seus resultados.

Assim, optou-se por realizar a pesquisa dos termos apenas nos textos completos de artigos, utilizando somente a base da ACM Digital Library, por ser esta das três bases pesquisadas, aquela em que a pesquisa dos termos retornava o maior número de resultados. Também decidiu-se considerar apenas as publicações de pesquisas da área de computação, publicadas nos anos de 2015 e 2016, focando nas pesquisas mais recentes da área que reportavam a utilização do método.

Sendo a ACM Digital Library um dinâmico repositório internacional, os critérios de inclusão definidos foram: o artigo deveria estar escrito em inglês, o artigo deveria explicitar o uso da etnografia, e o artigo deveria responder a pelo menos $50 \%$ das questões específicas de pesquisa (ao menos 3 QE's). O critério de exclusão estabelecido: no caso de artigos similares publicados em mais de uma fonte, seria considerado o mais recente ou o mais completo.

O processo de seleção de artigos se baseou no uso de quatro filtros, na ordem apresentada: pesquisa pela string de pesquisa em artigos publicados pela ACM Digital Library entre 2015 e 2016, análise do título, leitura rápida do artigo para verificar se abordava explicitamente o método de etnografia (respondendo de fato à questão de pesquisa QP), leitura detalhada do artigo para verificar se respondia a $50 \%$ das questões específicas de pesquisa (ao menos 3 QE's).

As dúvidas identificadas durante o desenvolvimento deste estudo foram debatidas e sanadas pelos autores deste trabalho em reuniões semanais, presenciais e/ou virtuais.

\section{Resultados}

O planejamento desta RSL teve início em agosto de 2016, a pesquisa piloto em bases de dados nacionais e internacionais. Foram pesquisados artigos publicados a partir de janeiro de 2015. A pesquisa na base de dados selecionada foi realizada em setembro de 2016 , e atualizada em março de 2017, para incluir os artigos publicados no período de setembro a dezembro de 2016.

Foram aplicados os filtros descritos na Seção 3. Na primeira etapa foram retornados 212 artigos publicados na base da ACM Digital Library. Após uma leitura rápida dos artigos com o 
objetivo de aplicar os critérios de inclusão da Seção 3, foram selecionados os artigos que respondiam a pelo menos $50 \%$ das questões de pesquisa e abordavam a temática educação.

A Tabela 1 sumariza a quantidade de artigos resultante em cada etapa da RSL após a aplicação de cada filtro, em sua última linha apresenta o número de artigos analisados neste artigo.

Tabela 1 - Resultado da etapa de aplicação dos filtros da RSL nos resultados da ACM Digital Library

\begin{tabular}{|c|c|}
\hline Etapa & Num. Artigos \\
\hline Pesquisa Inicial & 212 \\
\hline Análise de Títulos & 205 \\
\hline Leitura Rápida & 76 \\
\hline Artigos de Educação & 40 \\
\hline Leitura Detalhada & 35 \\
\hline
\end{tabular}

Fonte: Os autores.

\subsection{Qual o objetivo da pesquisa?}

Como apresentado na Seção 2, a etnografia é uma abordagem metodológica teórica que utiliza a investigação empírica para apoiar a pesquisa qualitativa em diversos contextos sociais. Os resultados desta RSL retornaram, por exemplo, o trabalho de Martinviita et al. (2015) que relata um estudo de caso que explora o cenário social por meio de um novo serviço on-line que tem como objetivo o aumento motivacional do estudo na educação profissional de jovens entre 16 e 18 anos. Edwards et al. (2016) apresentam a utilização do Facebook para apoiar a socialização e a aprendizagem em pares em uma universidade. A pesquisa de Giles e Linden (2015) apresenta um ambiente criado para que as pessoas possam aprender umas com as outras. Halloluwa et al. (2016) desenvolveram um estudo para entender como estudantes e professores usam a gamificação a partir de aplicativos móveis para aprender matemática na sala de aula.

Como pode ser observado na Tabela 2, 42\% dos 35 trabalhos selecionados na RSL apresentam questões relacionadas à compreensão como foco. Isso pode ser observado tanto na pesquisa de Mudliar e Rangaswamy (2015), que busca compreender a vida dos alunos e as escolhas de aprendizagem que fazem durante a faculdade, quanto na pesquisa de Pantic et al. (2016), que procura compreender o caminho percorrido por uma aluna novata para aprender programação.

Tabela 2 - Verbos identificados com maior frequência nos objetivos das pesquisas selecionadas na RSL

\begin{tabular}{|c|c|}
\hline Verbo & Num. Artigos \\
\hline Compreender & 13 \\
\hline Explorar & 5 \\
\hline Estudar & 5 \\
\hline
\end{tabular}




\begin{tabular}{|c|c|}
\hline Investigar & 5 \\
\hline
\end{tabular}

Fonte: Os autores

Termos como a "investigação" e a "exploração" também apareceram como objetivos dos estudos analisados. Pode se ver visto respectivamente nos trabalhos de Lupfer et al. (2016) que investiga as práticas criativas dos alunos de vários cursos para descobrir uma linguagem visual padrão, e no trabalho de Ahmed et al. (2015) que explora o conhecimento explícito, tático e social, proporcionando a prática e a experiência em reparos de telefones celulares nos mercados de Bangladesh.

Foi encontrado também o uso da etnografia para fins de avaliação como a pesquisa de Noble (2015), que avalia dados observacionais obtidos a partir de um estudo de caso em um ambiente de treinamento médico de hemodiálise em casa.

\subsection{Qual o período de investigação?}

Como pode ser observado na Tabela 3 a maior concentração de pesquisas teve tempo de duração compreendido entre um mês e até seis meses. Porém, também foi encontrado um relato de pesquisa de apenas algumas horas de duração (KAZIUNAS et al., 2015). Também foram identificados relatos de pesquisas que reportam anos de duração, como o de Meintjes \& Schelhowe (2016), que durou 4 anos, e o de Tan et al. (2016), que foi desenvolvida durante cinco anos.

Tabela 3 - Tempo de duração das investigações nas pesquisas selecionadas na RSL

\begin{tabular}{|c|c|}
\hline Período & Num. Artigos \\
\hline Até 1 dia & 1 \\
\hline De 1 dia a 1 mês & 5 \\
\hline De 1 a 6 meses & 10 \\
\hline De 6 meses a 1 ano & 8 \\
\hline Mais de 1 ano & 7 \\
\hline Não informado & 4 \\
\hline
\end{tabular}

Fonte: Os autores

\subsection{Qual o tamanho da amostra?}

A Tabela 4 resume os resultados levantados sobre o tamanho das amostras em cada pesquisa. Pode-se observar que na maior parte dos estudos avaliados o tamanho das amostras envolvidas variou de 6 a 50 participantes. Na sequência, estão aqueles estudos com amostras de até 150 participantes, seguido de perto pelos estudos desenvolvidos com amostras pequenas, com até cinco participantes. Apenas, dois dos estudos não forneceram informações efetivas sobre as amostras utilizadas (PRIOR; FERGUSON; LEANEY, 2016) e (AMES, 2015). 
Tabela 4 - Tamanho das amostras identificadas das investigações nas pesquisas selecionadas na RSL

\begin{tabular}{|c|c|}
\hline Tam. Da Amostra & Num. Artigos \\
\hline Com até 5 & 5 \\
\hline De 6 a 50 & 17 \\
\hline De 51 a 150 & 7 \\
\hline Mais de 150 & 4 \\
\hline Não informado & 2 \\
\hline
\end{tabular}

Fonte: Os autores

Outro aspecto identificado nessa avaliação das amostras foi que em dois estudos, observaram-se divergências relacionadas à quantidade de participantes. No primeiro caso os investigadores reportaram a redução do número de participantes na amostra após o estudo já ter sido iniciado (THERIAS; BIRD; MARSHALL, 2015). No segundo caso, a descrição da coleta de dados nos sugere alguma imprecisão da amostragem, já que os autores reportaram um número exato de pessoas entrevistadas formalmente, sem efetivamente contabilizar o número de pessoas que foram entrevistadas de maneira informal (WAYCOTT et al., 2016).

Cabe também destacar que quatro estudos utilizaram amostras com número de participantes superior a 150, algo que é considerado incomum para estudos qualitativos (AHMED; JACKSON; RIFAT, 2015; RONCO-LÓPEZ; ECHEGARAY-EIZAGUIRRE; PEÑAFIEL SÁIZ, 2016; CUTRELL et al., 2015; LUPFER et al., 2016).

\subsection{Quais foram os procedimentos adotados para coleta de dados?}

Um dos instrumentos de pesquisa mais utilizados foi a observação. Para melhor análise posterior dos dados, o período de observação é registrado em anotações e gravado (filme, fotografias e gravação de voz) (KNOBELSDORF \& FREDE, 2016). Por sua vez, as anotações podem considerar aspectos diversos, além da fala, tais como expressão facial, gestos, movimentos do corpo, bem como o momento em que cada um desses aspectos ocorreu, para melhor sincronização entre as anotações e as gravações (SADIK, 2015). Utilizando recursos digitais, a coleta de dados não necessariamente precisa ser presencial (etnografia virtual). Dados sobre o comportamento e ações dos participantes também podem ser obtidos a partir de arquivos de logs (trace ethnography) e análise de dados de comunidades virtuais (CAMPBELL et al., 2016; CUTRELL et al., 2015; MARTINVIITA; KUURE; LUOMA, 2015; MUDLIAR; RANGASWAMY, 2015; MUGAR et al., 2015; WAYCOTT et al., 2016).

O observador pode atuar como participante ou não. Em Cutrell et al. (2015), a observação foi realizada com o pesquisador fazendo anotações sentado no fundo de uma sala de aula. Por sua vez, Mudliar e Rangaswamy (2015) optaram por se mudar para o dormitório da universidade para poder conhecer melhor o cotidiano dos estudantes e suas interações, facilitando o registro de atividades e conversas informais (observação em profundidade). O observador também pode atuar como participante, o que é um método aceito na etnografia, porque, na verdade "toda 
etnografia representa as perspectivas do(s) etnógrafos(s)" (LINDER et al., 2015). Também como observadores participantes, durante seis meses, Mugar et al. (2015) realizaram atividades de alunos e "gastaram de uma a três horas semanais visitando o site do curso, participando e observando durante cada visita". Além disso, a observação pode se dar com ou sem a interferência do pesquisador (KNOBELSDORF; FREDE, 2016; CUTRELL et al., 2015). Um exemplo de interferência ocorre quando o pesquisador periodicamente encoraja os participantes a externalizarem suas ideias (MEINTJES; SCHELHOWE, 2016).

A observação não necessita ser ininterrupta: Yerousis et al. (2015) faziam visitas semanais a cada um dos clubes envolvidos na pesquisa; Cutrell et al. (2015) fizeram duas visitas a cada escola envolvida; Sadik (2015) realizou quatro sessões de 2 a 3 horas de observação; e, Griffin, Pirmann \& Gray (2016) realizaram 15 sessões de 90 minutos.

Em associação à observação, é comum conduzir entrevistas para completar e verificar informações (SOBEL; O'LEARY; KIENTZ, 2015). A entrevista pode ser realizada antes ou após as observações, ou antes e após (HALLOLUWA et al., 2016; WAYCOTT et al., 2016). As entrevistas que foram detalhadas têm, geralmente, duração aproximada de 60 minutos. Houve relato de casos de terem durado apenas 30 minutos (SADIK, 2015). O protocolo de entrevista mais utilizado foi o semi-estruturado, como em Sadik (2015) e Ssozi-Mugarura; Blake; Rivett (2016). Therias et al. (2015) optaram por realizar dois tipos de entrevistas: semi-estruturada e nãoestruturada. Conversas informais também foram registradas (YEROUSIS et al., 2015; WAYCOTT et al., 2016).

Alguns autores explicitaram o processo de rapport, que se trata do estabelecimento de um relacionamento de confiança e respeito mútuo, o que gera mais tranquilidade para os participantes (MUDLIAR; RANGASWAMY, 2015). Em Therias et al. (2015), o pesquisador foi apresentado como alguém que estaria em sala para observar e ajudar, quando necessário. Edwards et al. (2016) optaram por definir como entrevistador alguém que já era conhecido e respeitado pela comunidade objeto do estudo.

É importante, ainda, que o pesquisador conheça e compreenda o grupo ou comunidade que irá observar, antes de iniciar suas atividades de pesquisa (CAMPBELL et al., 2016; SSOZIMUGARURA; BLAKE; RIVETT, 2016). Isso permite redefinir o processo de coleta de dados, a forma de abordagem e a seleção dos participantes. Por isso, alguns autores optaram por participar de 20 sessões de tutorial (KNOBELSDORF; FREDE, 2016), enquanto outros participaram em cursos para ter mais contato com o público-alvo de seu trabalho (AHMED; JACKSON; RIFAT, 2015; NOBLE, 2015; MAO; BLACKWELL; LUKATE; GOOD, 2016). Também houve caso da necessidade de realizar as atividades esperadas pela comunidade virtual, como escrever uma fanfiction (CAMPBELL et al., 2016), para que os pesquisadores pudessem se integrar a ela. Uma prática comum é a organização de workshops para facilitar o processo de discussão entre e com os participantes (GILES; LINDEN, 2015; YEROUSIS et al., 2015).

As atividades de uma pesquisa etnográfica não precisam se limitar àquelas formalmente planejadas. Por exemplo, os participantes podem interagir regularmente com o pesquisador e compartilhar mensagens do cotidiano (JONES; PAL, 2015), por email, aplicativos diversos de mensagem ou por telefone (WAYCOTT et al., 2016). 
A etnografia também pode ser combinada outras metodologias pesquisa que utilizam atividades coletivas próprias como a pesquisa-ação (PRIOR; FERGUSON; LEANEY, 2016; YEROUSIS et al., 2015), design participativo (SSOZI-MUGARURA; BLAKE; RIVETT, 2016) e incluir a realização de testes com usuários (ROCHA et al., 2015). Com relação ao uso de atividades de design para compreender melhor as "perspectivas, desejos e necessidades" do público-alvo, foi identificado a aplicação do método de design ethnography com crianças (SOBEL; O'LEARY; KIENTZ, 2015). Além desse método e da etnografia online, foram também utilizados: autoetnografia, derivada da participação ativa do pesquisador (NOBLE, 2015); etnografia rápida, que "se caracteriza pelo uso de múltiplos métodos para coletar um conjunto rico de dados em um curto período de tempo" (THERIAS; BIRD; MARSHALL, 2015) e uma adaptação da etnografia visual (LUPFER et al., 2016) para estudar objetos digitais multimídia disponíveis na Web e desenvolver uma teoria visual fundamentada.

Diversas estratégias podem ser adotadas para reduzir contradições e erros de interpretação dos dados coletados, tais como conversas periódicas entre os pesquisadores (TAN et al., 2016) e realização de workshops com apresentação e avaliação dos dados coletados com os participantes (SSOZI-MUGARURA; BLAKE; RIVETT, 2016). Alguns autores citam explicitamente a triangulação (KAZIUNAS et al., 2015), como melhor forma de garantia da qualidade dos dados coletados.

Definições formais sobre os procedimentos e instrumentos identificados nas pesquisas analisadas podem ser consultadas em Hammersley; Atkinson (2007) e CRESWELL (2002). Mais detalhes sobre os diversos instrumentos de pesquisa e fontes de dados utilizados são apresentados na subseção 4.4.1.

\subsubsection{Quais foram os instrumentos de coleta de dados adotados?}

Os instrumentos de pesquisa mais utilizados foram a entrevista e a observação. De acordo com a seção 2, um dos instrumentos muito utilizados em pesquisas etnográficas são os diários, entretanto somente um trabalho utilizou este instrumento de pesquisa [BAHARIN et al. 2015]. 0 grupo focal, assim como os questionários, foi utilizado em três trabalhos, em conjunto com entrevistas, para aumentar o envolvimento dos participantes [KAZIUNAS et al. 2015; SSOZIMUGARURA et al. 2016; Therias et al. 2015].

Para coleta de informações, foram utilizadas pelos pesquisadores 10 tipos de fontes diferentes. As anotações foram as mais utilizadas nos artigos selecionados. $O$ trabalho de Giles and Van der Linden (2015) foi o artigo que explorou o maior número de fontes, 5 no total: anotações, vídeos, fotos e áudio. As tabelas 5 e 6 resumem os resultados relacionados aos instrumentos de pesquisa e as fontes de coleta de dados mais utilizadas nos artigos analisados, respectivamente. A fonte de pesquisa "Plataformas Digitais" na tabela 6 faz referência aos artigos que mencionaram como fonte de dados o uso de: sites, blogs, mídias sociais e formulário digitais. O trabalho de Partogi and JafariNaimi (2016) fez uso de plataformas digitais. 
Tabela 5 - Instrumentos de Pesquisa utilizados que foram identificados nas pesquisas selecionadas na RSL

\begin{tabular}{|c|c|}
\hline Instrumentos & Num. Artigos \\
\hline Entrevistas & 30 \\
\hline Observação & 29 \\
\hline Grupo Focal & 3 \\
\hline Diário & 1 \\
\hline Questionário & 3 \\
\hline
\end{tabular}

Fonte: Os autores

Tabela 6 - Fontes de dados mais utilizadas que foram identificadas nas pesquisas selecionadas na RSL.

\begin{tabular}{|c|c|}
\hline Fonte de Dados & Num. Artigos \\
\hline Anotações & 11 \\
\hline Survey & 4 \\
\hline Vídeo & 4 \\
\hline Fotos & 6 \\
\hline Audio & 3 \\
\hline Plataformas Digitais & 24 \\
\hline
\end{tabular}

Fonte: Os autores

\subsection{Como a análise de dados foi realizada?}

Diferentes métodos foram utilizados pelos autores para análise dos dados coletados. Tan et al. (2016) afirmam terem utilizado um framework conceitual, que inclui a leitura de transcrições de entrevistas e conversas, momentos de desenho crítico a análises de notas de campo. Kaziunas et al. (2015), Campbell et al. (2016) e Linder et al. (2015) utilizaram teoria fundamentada. Pantic et al. (2016) empregou uma análise combinada, utilizando dados observacionais, estudos de transcrições de reuniões de debriefing e uma abordagem de teoria fundamentada para notas de campo. Em Griffin et al. (2016) foi utilizada codificação, análise de estudo de caso e análise cruzada de casos. Sadik (2015) utilizou análise de campo de significado e uma abordagem de codificação, enquanto Lupfer et al. (2016) utilizaram teoria visual fundamentada, codificação focada e métrica de ideação de fluência.

Prior et al. (2016) utilizaram abordagem iterativa e análise de interação. Sobel et al. (2015), por sua vez, analisaram os dados de sua pesquisa utilizando abordagem iterativa e codificação qualitativa, ambas de forma indutiva e dedutiva. Edwards et al. (2016) realizaram análise qualitativa de conteúdo e empregaram o modelo de estágios de Tuckman. Já Jones \& Pal (2015) delimitaram temas utilizando codificação aberta e memorandos reflexivos para relacionar notas de campos com as narrativas dos participantes. A análise temática foi a única técnica utilizada por Noble (2015) e por Mao et al. (2016). Baharin et al. (2015) utilizaram uma abordagem por 
eles denominada como exemplos perspicazes qualitativos, Mudliar \& Rangaswamy (2015), um método comparativo constante, e finalmente Meintjes \& Schelhowe (2016), utilizaram análise de interação.

\section{Discussão}

A presente RSL teve como objetivo avaliar como a comunidade de pesquisadores da área de Informática na Educação de um modo geral faz uso do método etnográfico em suas pesquisas. Com o trabalho desenvolvido, foi possível identificar uma diversidade de situações de uso e apontar detalhes da aplicação da etnografia nas pesquisas de Informática na Educação no contexto internacional, conforme foi apresentado pelos resultados de análise dos 35 artigos selecionados da base da ACM Digital Library. É importante ressaltar que durante o processo não foi encontrada na literatura analisada outra RSL que contemplasse o mesmo objetivo que o desta pesquisa.

As análises dos materiais no âmbito da pesquisa internacional no domínio específico da Informática na Educação, apontam para o uso do método etnográfico de uma forma mais regular do que foi identificado no contexto nacional, dado o reduzido número de trabalhos identificados, o que tornou inviável a utilização de repositórios nacionais, como especificado na seção 3. Percebe-se uma utilização mais efetiva da etnografia pela comunidade internacional de pesquisadores, reconhecendo o mérito desta abordagem como uma forma natural para avaliar o impacto nos fatores humanos dos produtos tecnológicos ou dos procedimentos de introdução da tecnologia no contexto educacional.

De um modo geral, no processo de seleção e avaliação do material, quando da aplicação dos filtros definidos na Seção 3, também se percebeu uma maior apropriação dos métodos qualitativos em pesquisas de fora do domínio humanístico, por áreas como a engenharia, tecnologia, design e marketing. Uma das justificativas da amplificação do uso do método na área de ciências exatas seria o maior entendimento do mérito da abordagem etnográfica para melhor identificar características essenciais para o sucesso das pesquisas direcionadas a um público-alvo ou para atender um contexto especifico: desenvolver um produto, avaliar o impacto do uso de um equipamento, reformular um procedimento, etc. Igualmente foi identificada essa mesma percepção de assertividade da abordagem etnográfica, isolada ou em composição de um método misto, nos objetivos de pesquisa dos artigos selecionados. Principalmente nas pesquisas que avaliavam o impacto das tecnologias no contexto social e/ou educacional, a partir de uma análise mais focada no fator humano envolvido.

Consideramos importante ressaltar que só foi possível identificar uma utilização relevante da etnografia em estudos relativos à Informática na Educação apenas no âmbito internacional, já que o mesmo não pode ser verificado no âmbito da pesquisa nacional. Consideramos igualmente importante a identificação do elevado emprego da abordagem etnográfica nos últimos anos em pesquisas das ciências exatas, como engenharia, computação e tecnologia, dado o número de trabalhos retornados nos repositórios internacionais ao se buscar pelas strings de pesquisa. 0 volume de pesquisas retornadas na base selecionada foi elevado (212 resultados), ainda que 
deste total apenas 35 estivessem de fato no domínio da Informática na Educação, e que efetivamente indicavam a utilização da etnografia como abordagem de pesquisa, a medida em que as etapas de refino de pesquisa foram sendo concluídas.

Entretanto, esse resultado pode ser considerado significativo, se compararmos os resultados obtidos na pesquisa realizada nos repositórios nacionais. Nesse caso a busca retornou apenas 4 resultados associando a etnografia a trabalhos desenvolvidos e publicados pela comunidade de Informática na Educação no Brasil, sendo que apenas 1 deles foi publicado dentro do período de investigação definido para esta RSL, 2015 e 2016. Pode se indicar este como um fator limitante para os objetivos de pesquisa, o que torna ainda mais relevantes os resultados desta RSL para os pesquisadores nacionais. Estes resultados também apontam como relevante a realização de uma análise mais aprofundada sobre as causas da baixa utilização do método etnográfico na pesquisa em Informática na Educação no contexto nacional.

Outrossim, no rol de limitações de pesquisa identificados, foi observado também que entre alguns dos trabalhos selecionados encontramos um grau moderado de incongruências sobre aspectos conceituais da utilização do método etnográfico. Por exemplo, durante as fases de leitura foi identificado a ocorrência de dois tipos de situações: (i) casos em que nos artigos selecionados, o termo pesquisado aparecia nas palavras-chaves e/ou na indicação de área de domínio, mas o texto completo do artigo não apresentava detalhes descritivos da aplicação do método efetivamente; e ainda, (ii) casos em que era possível identificar elementos de pesquisa compatíveis com abordagem etnográfica, contudo, a única citação ao termo de pesquisa se encontrava apenas na seção de trabalhos relacionados e/ou nas referências bibliográficas do texto completo. O fato de situações como essas terem sido identificadas podem ser compreendidos como uma indicação de que persiste ainda algum nível de confusão e/ou desinformação (e.g., misconceptions) por parte de alguns dos pesquisadores internacionais a respeito do uso de elementos essenciais do método de pesquisa etnográfica.

Outra limitação importante associada ao processo de desenvolvimento desta RSL foram as dificuldades encontradas para identificar informações precisas quanto: aos períodos de desenvolvimento do estudo etnográfico e da descrição da amostra. No primeiro caso, é preciso fazer uma distinção clara entre período de desenvolvimento do estudo etnográfico, uma vez que esse conceito difere do período que compreende as datas em que as observações foram realizadas, os dados coletados e do tempo empregado para analisar os dados obtidos. No segundo caso, também consideramos relevante que os autores fiquem atentos no detalhamento descritivo das amostras, estabelecendo uma clara diferenciação entre a descrição que fazem do público-alvo da pesquisa, da descrição que fazem do conjunto de indivíduos que efetivamente participaram da amostra pesquisada. Ainda assim, seria possível associar uma parte dessas dificuldades com a falta de detalhamento na descrição desses aspectos da pesquisa por parte dos autores dos artigos avaliados. 


\section{Conclusões}

Considerando a relevância da etnografia para pesquisas cujo foco está no fator humano, os resultados deste trabalho podem contribuir para que grupos de pesquisa em Informática na Educação no Brasil possam ampliar a utilização do método etnográfico. Uma maior apropriação da abordagem etnográfica poderia aumentar as possibilidades de pesquisa na área de Informática na Educação e, por consequência, qualificar a produção científica nacional, o que beneficiaria sobremaneira a prática educacional em seus vários níveis e modalidades.

Como um dos resultados deste trabalho, o objetivo de oferecer aos pesquisadores brasileiros uma fonte de consulta foi cumprido. Estes poderão mais facilmente buscar informações sobre como o método etnográfico tem sido empregado nas pesquisas da área no contexto internacional, de forma a melhor orientar o planejamento de suas pesquisas no contexto nacional.

Ao longo do texto, este trabalho identifica diversos aspectos conceituais e elementos de pesquisas próprios da abordagem etnográfica, descrevendo a maneira como estes foram utilizados nas pesquisas analisadas. Os pesquisadores nacionais poderão consultar, por exemplo, nas referências quais os artigos que relatam um período de tempo de pesquisa curto ou um número muito grande ou muito pequeno de participantes. Isso permite que seja possível avaliar antecipadamente o quanto os contextos de pesquisas apresentados são compatíveis com suas necessidades e realidades, de maneira que seja possível avaliar com mais confiança a admissibilidade e a conveniência do uso da abordagem etnográfica para sua própria pesquisa. Além disso, também poderão encontrar sugestões para definição de atividades e para a seleção de instrumentos de coleta de dados, bem como de métodos e técnicas utilizados para análise desses dados.

Como trabalhos futuros sugere-se ampliar o escopo do presente estudo para compreender um período maior de tempo do que os dois anos aqui pesquisados e adicionar as publicações das outras duas bases de pesquisa. Também se considera relevante incluir uma análise mais detalhada dos artigos citados nas referências dos estudos primários selecionados.

\section{Agradecimentos}

Os autores agradecem à Coordenação de Aperfeiçoamento de Pessoal de Nível Superior (CAPES) e a Pontifícia Universidade Católica de Minas Gerais (PUC-Minas) pelo apoio financeiro concedido aos pesquisadores envolvidos, na forma de bolsa de doutorado e de estágio pósdoutoral PNPD, durante o desenvolvimento deste trabalho.

\section{Referências Bibliográficas}

AHMED, S. I.; JACKSON, S. J.; RIFAT, M. R. Learning to fix: Knowledge, collaboration and mobile phone repair in Dhaka, Bangladesh. In: Proceedings of the Seventh International Conference on Information and Communication Technologies and Development. New York, NY, USA: ACM, 2015. (ICTD '15), p. 4:1-4:10. ISBN 978-1-4503-3163-0. http://doi.acm.org/10.1145/2737856.2738018i. 
AMES, M. G. Charismatic technology. In: Proceedings of The Fifth Decennial Aarhus Conference on Critical Alternatives. Aarhus University Press, 2015. (AA '15), p. 109-120.

http://dx.doi.org/10.7146/aahcc.v1i1.21199i.

BAHARIN, H.; VILLER, S.; RINTEL, S. Sonicair: Supporting independent living with reciprocal ambient audio awareness. ACM Trans. Comput.-Hum. Interact., ACM, New York, NY, USA, v. 22, n. 4, p. 18:1-18:23, jul. 2015. ISSN 1073-0516. http://doi.acm.org/10.1145/2754165i.

CASAS, T. H. P. Computer Science in the Education: The Vision of the teachers. In Revista Brasileira de Informática na Educação. Vol. 12, No. 2, 2004. Disponível em http://www.brie.org/pub/index.php/rbie/article/view/2190.DOI:http://dx.doi.org/10.5753/rbie.2004.12.2.88-89

CAMPBELL, J. et al. Thousands of positive reviews: Distributed mentoring in online fan communities. In: Proceedings of the 19th ACM Conference on Computer-Supported Cooperative Work \& Social Computing. New York, NY, USA: ACM, 2016. (CSCW '16), pp. 691-704. ISBN 978-1-4503-3592-8. http://doi.acm.org/10.1145/2818048.2819934i.

CRESWELL, J. W. Educational research: Planning, conducting, and evaluating quantitative. [S.I.]: Prentice Hall Upper Saddle River, NJ, 2002.

CUTRELL, E. et al. Blended learning in Indian colleges with massively empowered classroom. In: Proceedings of the Second (2015) ACM Conference on Learning @ Scale. New York, NY, USA: ACM, 2015. (L@S '15), p. 47-56. ISBN 978-1-4503-3411-2. http://doi.acm.org/10.1145/2724660.2724666i.

EDWARDS, M.; DARWENT, D.; IRONS, C. That blasted facebook page: Supporting trainee-teachers professional learning through social media. SIGCAS Comput. Soc., ACM, New York, NY, USA, v. 45, n. 3, p. 420-426, jan. 2016. ISSN 0095-2737. http://doi.acm.org/10.1145/2874239.2874301i.

GILES, E.; LINDEN, J. van der. Imagining future technologies: Etextile weaving workshops with blind and visually impaired people. In: Proceedings of the 2015 ACM SIGCHI Conference on Creativity and Cognition. New York, NY, USA: ACM, 2015. (C\&\#38;C '15), p. 3-12. ISBN 978-1-4503-3598-0. http://doi.acm.org/10.1145/2757226.2757247i.

GRIFFIN, J.; PIRMANN, T.; GRAY, B. Two teachers, two perspectives on cs principles. In: Proceedings of the 47th ACM Technical Symposium on Computing Science Education. New York, NY, USA: ACM, 2016. (SIGCSE '16), p. 461-466. ISBN978-1-4503-3685-7. http://doi.acm.org/10.1145/2839509.2844630i.

HALLOLUWA, T. et al. Gamifying mathematics for primary students in rural Sri Lanka. In: Proceedings of the 9th Nordic Conference on Human-Computer Interaction. New York, NY, USA: ACM, 2016. (NordiCHI '16), p. 62:1-62:4. ISBN 978-1-4503-4763-1. http://doi.acm.org/10.1145/2971485.2971522i.

HAMMERSLEY, M.; ATKINSON, P. Ethnography: Principles in practice. [S.I.]: Routledge, 2007.

JONES, J.; PAL, J. Counteracting dampeners: Understanding technology-amplified capabilities of people with disabilities in Sierra Leone. In: Proceedings of the Seventh International Conference on Information and Communication Technologies and Development. New York, NY, USA: ACM, 2015. (ICTD '15), p. 6:16:10. ISBN978-1-4503-3163-0. http://doi.acm.org/10.1145/2737856.2738025i.

KAZIUNAS, E. et al. Transition and reflection in the use of health information: The case of pediatric bone marrow transplant caregivers. In: Proceedings of the 18th ACM Conference on Computer Supported Cooperative Work 38; Social Computing. New York, NY, USA: ACM, 2015. (CSCW '15), p. 1763-1774. ISBN 978-1-4503-2922-4. http://doi.acm.org/10.1145/2675133.2675276i.

KITCHENHAM, B. A. Guidelines for performing Systematic Literature Reviews in Software Engineering, Version 2.3, EBSE-2007-01. Durham, UK, 2007.

KNOBELSDORF, M.; FREDE, C. Analyzing student practices in theory of computation in light of distributed cognition theory. In: Proceedings of the 2016 ACM Conference on International Computing Education Research. New York, NY, USA: ACM, 2016. (ICER '16), p. 73-81. ISBN 978-1-4503-4449-4. http://doi.acm.org.ez93.periodicos.capes.gov.br/10.1145/2960310.2960331i. 
LINDER, R. et al. Beyond slideware: How a free-form presentation medium stimulates free-form thinking in the classroom. In: Proceedings of the 2015 ACM SIGCHI Conference on Creativity and Cognition. New York, NY, USA: ACM, 2015. (C\&\#38;C'15), p. 285-294. ISBN 978-1-4503-3598-0.

http://doi.acm.org/10.1145/2757226.2757251i.

LUPFER, N. et al. Patterns of free-form curation: Visual thinking with web content. In: Proceedings of the 2016 ACM on Multimedia Conference. New York, NY, USA: ACM, 2016. (MM '16), p. 12-21. ISBN 978-14503-3603-1. http://doi.acm.org/10.1145/2964284.2964303i.

MAO, M. et al. Supporting retirement socially and musically by technology: An ethnographic study of local community musicians. In: Proceedings of the $2016 \mathrm{CHI}$ Conference Extended Abstracts on Human Factors in Computing Systems. New York, NY, USA: ACM, 2016. (CHI EA '16), p. 2886-2892. ISBN 978-1-45034082-3. http://doi.acm.org/10.1145/2851581.2892285i.

MARTINVIITA, A.; KUURE, L.; LUOMA, P. Do we speak the same language? Design goals and culture clashes in an online forum for young people. In: Proceedings of the 7th International Conference on Communities and Technologies. New York, NY, USA: ACM, 2015. (C\&\#38;T'15), p. 69-78. ISBN 978-14503-3460-0. http://doi.acm.org.ez93.periodicos.capes.gov.br/10.1145/2768545.2768550i.

MEINTJES, R.; SCHELHOWE, H. Inclusive interactives: The transformative potential of making and using craft-tech social objects together in an after-school centre. In: Proceedings of the 15th International Conference on Interaction Design and Children. New York, NY, USA: ACM, 2016. (IDC '16), p. 89-100. ISBN 978-1-4503-4313-8. http://doi.acm.org/10.1145/2930674.2930685i.

MUDLIAR, P.; RANGASWAMY, N. Offline strangers, online friends: Bridging classroom gender segregation with WhatsAPP. In: Proceedings of the 33rd Annual ACM Conference on Human Factors in Computing Systems. New York, NY, USA: ACM, 2015. (CHI '15), p. 3799-3808. ISBN 978-1-4503-3145-6. http://doi.acm.org/10.1145/2702123.2702533i.

MUGAR, G. et al. Being present in online communities: Learning in citizen science. In: Proceedings of the 7th International Conference on Communities and Technologies. New York, NY, USA: ACM, 2015. (C\&\#38;T '15), p. 129-138. ISBN 978-1-4503-3460-0. http://doi.acm.org/10.1145/2768545.2768555i.

NOBLE, P. J. Resilience ex machina: Learning a complex medical device for haemodialysis self-treatment. In: Proceedings of the 33rd Annual ACM Conference on Human Factors in Computing Systems. New York, NY, USA: ACM, 2015. (CHI '15), p. 4147-4150. ISBN 978-1-4503-3145-6. http://doi.acm.org/10.1145/2702123.2702348i.

PANTIC, K.; FIELDS, D. A.; QUIRKE, L. Studying situated learning in a constructionist programming camp: A multimethod microgenetic analysis of one girl's learning pathway. In: Proceedings of the 15th International Conference on Interaction Design and Children. New York, NY, USA: ACM, 2016. (IDC '16), p. 428-439. ISBN 978-1-4503-4313-8. http://doi.acm.org/10.1145/2930674.2930725i.

PARTOGI, M.; JAFARINAIMI, N. Fostering organizational change through co-designing collaborative media. In: Proceedings of the 19th International Conference on Supporting Group Work. New York, NY, USA: ACM, 2016. (GROUP '16), p. 441-444. ISBN 978-1-4503-4276-6.

http://doi.acm.org/10.1145/2957276.2996289i.

PRIOR, J.; FERGUSON, S.; LEANEY, J. Reflection is hard: Teaching and learning reflective practice in a software studio. In: Proceedings of the Australasian Computer Science Week Multiconference. New York, NY, USA: ACM, 2016. (ACSW '16), p. 7:1-7:8. ISBN 978-1-4503-4042-7. http://doi.acm.org/10.1145/2843043.2843346i.

ROCHA, T. et al. Performing universal tasks on the web: Interaction with digital content by people with intellectual disabilities. In: Proceedings of the XVI International Conference on Human Computer Interaction. New York, NY, USA: ACM, 2015. (Interaccin'15), p. 30:1-30:7. ISBN 978-1-4503-3463-1.: http://doi.acm.org/10.1145/2829875.2829897i.

RONCO-LÓPEZ, M.; ECHEGARAY-EIZAGUIRRE, L.; SÁIZ, C. PEÑAFIEL. The concept of health and the evaluation of internet as a health information search tool of the youth of the basque country and navarra. In: Proceedings of the Fourth International Conference on Technological Ecosystems for Enhancing 
Multiculturality. New York, NY, USA: ACM, 2016. (TEEM '16), p. 1053-1058. ISBN 978-1-4503-4747-1. http://doi.acm.org/10.1145/3012430.3012646i.

SADIK, O. Encouraging women to become cs teachers. In: Proceedings of the Third Conference on GenderIT. New York, NY, USA: ACM, 2015. (GenderIT '15), p. 57-61. ISBN 978-1-4503-3596-6. http://doi.acm.org/10.1145/2807565. 2807715i.

SOBEL, K.; O'LEARY, K.; KIENTZ, J. A. Maximizing children's opportunities with inclusive play: Considerations for interactive technology design. In: Proceedings of the 14th International Conference on Interaction Design and Children. New York, NY, USA: ACM, 2015. (IDC '15), p. 39-48. ISBN 978-1-45033590-4. http://doi.acm.org/10.1145/2771839.2771844i.

SSOZI-MUGARURA, F.; BLAKE, E.; RIVETT, U. Supporting community needs for rural water management through community-based co-design. In: Proceedings of the 14th Participatory Design Conference: Full Papers - Volume 1. New York, NY, USA: ACM, 2016. (PDC '16), p. 91-100. ISBN 978-1-4503-4046-5. http://doi.acm.org/10.1145/2940299.2940311i.

TAN, E. et al. Probing participatory partnerships: Equitably-consequential making by, for and with marginalized youth. In: Proceedings of the 6th Annual Conference on Creativity and Fabrication in Education. New York, NY, USA: ACM, 2016. (FabLearn'16), p. 1-8. ISBN 978-1-4503-4802-7. em: http://doi.acm.org/10.1145/3003397.3003398i.

THERIAS, E.; BIRD, J.; MARSHALL, P. Mas tecnologia, más cambio? Investigating an educational technology project in rural Peru. In: Proceedings of the 33rd Annual ACM Conference on Human Factors in Computing Systems. New York, NY, USA: ACM, 2015. (CHI '15), p. 447-456. ISBN 978-1-4503-3145-6. http://doi.acm.org/10.1145/2702123.2702595i.

TIMMERMANN, G. K.; GONZÁLEZ, F. Mediações que os professores e alunos estabelecem com o conteúdo da disciplina de Algoritmos de cursos superiores: estudo de caso. In Anais dos Workshops do Congresso Brasileiro de Informática na Educação. Vol. 5, No. 1, pp. 1295-1305, 2016. http://dx.doi.org/10.5753/cbie.wcbie.2016.1295.

VENTURA, P. P. B.; DE CASTRO FILHO, J. A. Relações sociais em comunidades virtuais de aprendizagem. In Anais do workshop de informática na escola 2010. WIE 2010. Vol. 1, No. 1, pp. 1235-1244, 2010. SBC. http://www.br-ie.org/pub/index.php/wie/article/view/2046.

WAYCOTT, J. et al. Not for me: Older adults choosing not to participate in a social isolation intervention. In: Proceedings of the 2016 CHI Conference on Human Factors in Computing Systems. New York, NY, USA: ACM, 2016. (CHI '16), p. 745-757. ISBN 978-1-4503-3362-7. http://doi.acm.org/10.1145/2858036.2858458i.

YEROUSIS, G. et al. Computer-enabled project spaces: Connecting with Palestinian refugees across camp boundaries. In: Proceedings of the 33rd Annual ACM, Conference on Human Factors in Computing Systems. New York, NY, USA: ACM, 2015. (CHI '15), p. 3749-3758. ISBN 978-1-4503-3145-6. http://doi.acm.org.ez93.periodicos.capes.gov.br/10.1145/2702123.2702283i.

Recebido em outubro de 2018.

Aprovado para publicação em maio de 2019.

\section{Scheila Wesley Martins}

Programa de Pós-Graduação Strictu Sensu em Informática - Pontifícia Universidade Católica de Minas Gerais - PUC/MINAS, Brasil, scheila.martins@sga.pucminas.br

\section{Daniel Eugênio Neves}

Programa de Pós-Graduação Strictu Sensu em Informática - Pontifícia Universidade Católica de Minas Gerais - PUC/MINAS, Brasil, danieleugenio.neves@gmail.com 


\section{Mônica da Consolação Machado}

Programa de Pós-Graduação Strictu Sensu em Informática - Pontifícia Universidade Católica de Minas Gerais

- PUC/MINAS, Brasil, monicacmachado@hotmail.com

\section{Ezequiel Mendes Duque}

Programa de Pós-Graduação Strictu Sensu em Informática - Pontifícia Universidade Católica de Minas Gerais

- PUC/MINAS, Brasil, ezequiel.duque@sga.pucminas.br

\section{Artur Martins Mol}

Programa de Pós-Graduação Strictu Sensu em Informática - Pontifícia Universidade Católica de Minas Gerais - PUC/MINAS, Brasil, amol@pucminas.br

\section{Lucila Ishitani}

Programa de Pós-Graduação Strictu Sensu em Informática - Pontifícia Universidade Católica de Minas Gerais

- PUC/MINAS, Brasil, lucila@pucminas.br

\section{Luana Giovani Noronha de Oliveira Santos}

Programa de Pós-Graduação Strictu Sensu em Informática - Pontifícia Universidade Católica de Minas Gerais

- PUC/MINAS, Brasil, luana.giovani@gmail.com 